\title{
Bayesian estimation for the exponential distribution based on generalized multiply Type-II hybrid censoring
}

\author{
Young Eun Jeon ${ }^{a}$, Suk-Bok Kang ${ }^{1, a}$ \\ ${ }^{a}$ Department of Statistics, Yeungnam University, Korea
}

\begin{abstract}
The multiply Type-II hybrid censoring scheme is disadvantaged by an experiment time that is too long. To overcome this limitation, we propose a generalized multiply Type-II hybrid censoring scheme. Some estimators of the scale parameter of the exponential distribution are derived under a generalized multiply Type-II hybrid censoring scheme. First, the maximum likelihood estimator of the scale parameter of the exponential distribution is obtained under the proposed censoring scheme. Second, we obtain the Bayes estimators under different loss functions with a noninformative prior and an informative prior. We approximate the Bayes estimators by Lindleys approximation and the Tierney-Kadane method since the posterior distributions obtained by the two priors are complicated. In addition, the Bayes estimators are obtained by using the Markov Chain Monte Carlo samples. Finally, all proposed estimators are compared in the sense of the mean squared error through the Monte Carlo simulation and applied to real data.
\end{abstract}

Keywords: Bayes estimator, exponential distribution, generalized multiply Type-II hybrid censoring, informative prior, loss function, maximum likelihood estimator, noninformative prior

\section{Introduction}

Consider an exponential distribution with the probability density function (pdf)

$$
f(x ; \sigma)=\frac{1}{\sigma} \exp \left(-\frac{x}{\sigma}\right)
$$

and the cumulative distribution function (cdf)

$$
F(x ; \sigma)=1-\exp \left(-\frac{x}{\sigma}\right), \quad x>0, \sigma>0 .
$$

The exponential distribution has been discussed by many researchers as the lifetime distribution. Kang and Park (2005) discussed the estimation for exponentiated exponential distribution based on multiply Type-II censoring scheme. Singh and Kumar (2007) provided the Bayes estimators of the exponential parameter under the multiply Type-II censoring scheme. Ganguly et al. (2012) studied the exact inference for the two-parameter exponential distribution under the Type-II hybrid censoring scheme. Childs et al. (2012) studied the exact distribution of the maximum likelihood estimators (MLEs) of the parameters and of the quantiles of two-parameter exponential distribution under the

\footnotetext{
${ }^{1}$ Corresponding author: Department of Statistics, Yeungnam University, 280 Daehak-ro, Gyeongsan, Gyeongbuk 38541 , Korea. E-mail: sbkang@yu.ac.kr
}

Published 31 July 2020 / journal homepage: http://csam.or.kr

(c) 2020 The Korean Statistical Society, and Korean International Statistical Society. All rights reserved. 
hybrid censoring scheme. Kang et al. (2012) studied the estimation of the entropy for a double exponential distribution based on the multiply Type-II censored samples. Kang et al. (2013) obtained the MLEs and some approximate MLEs of unknown parameters of the generalized exponential distribution when data are lower record values. Chan et al. (2015) studied the statistical inference of the two-parameter exponential distribution under the Type-II progressive hybrid censoring scheme. Cho et al. (2015) discussed the exact distribution of the MLE and the exact confidence interval for the parameter of the exponential distribution based on the generalized progressive hybrid censoring scheme.

Epstein (1954) introduced a Type-I hybrid censoring scheme in which the experiment is terminated at a random time $T^{*}=\min \left(X_{r: n}, T\right)$, where $X_{r: n}$ denotes the $r^{\text {th }}$ ordered failure time when the sample size is $n, r \in(1,2, \ldots, n)$ and $T \in(0, \infty)$ are pre-fixed. The Type-I hybrid censoring scheme has the disadvantage that very few units can be observed. To overcome this disadvantage of the TypeI hybrid censoring scheme, Childs et al. (2003) proposed a Type-II hybrid censoring scheme in which the experiment is terminated at a random time $T^{*}=\max \left(X_{r: n}, T\right)$. However, some units can be failed between two points of observation with exact times of failure of these units unobserved under the Type-II hybrid censoring scheme. For example, when we check the lifetime of people through the device, the device can turn off or break during the experimental progress, in which case we cannot check the exact time of some units failed during that duration. Therefore, Lee and Lee (2018) introduced the multiply Type-II hybrid censoring scheme. However, the multiply Type-II hybrid censoring scheme has the disadvantage of an excessively long experiment time, which necessitates a new censoring scheme.

In this paper, we propose a generalized multiply Type-II hybrid censoring scheme by referring to the method proposed by Chandrasekar et al. (2004) and also derive some estimators of the scale parameter for the exponential distribution under a proposed censoring scheme. In Section 2, we explain a generalized multiply Type-II hybrid censoring scheme with an MLE obtained under the proposed censoring scheme. In Section 3, some Bayes estimators are obtained by various priors and loss functions. For the prior distribution, a noninformative prior and an informative prior are considered. For the loss function, the squared error loss function (SELF), the linex loss function (LLF), and the general entropy loss function (GELF) are considered. We approximate the Bayes estimators by two methods (Lindley's approximation and the Tierney-Kadane (T-K) approximation) since the posterior distributions obtained by the two priors are complicated. We also obtain the Bayes estimators by the Markov Chain Monte Carlo (MCMC) samples. In Section 4, we compare the performance of the proposed estimators in the sense of the mean squared error (MSE) through the Monte Carlo simulation and apply the proposed estimators to real data. In Section 5, we provide the conclusion.

\section{Generalized multiply Type-II hybrid censoring}

Suppose the initial, middle and final observations are censored under the Type-II hybrid censoring scheme. Then, we have the following two cases under the multiply Type-II hybrid censoring scheme;

$$
\begin{aligned}
& \text { Case I : } X_{a_{1}: n}<X_{a_{2}: n}<\cdots<X_{a_{r}: n}<\cdots<X_{a_{d}: n}<T \\
& \text { Case II }: X_{a_{1}: n}<X_{a_{2}: n}<\cdots<X_{a_{d}: n}<T<X_{a_{d+1}: n}<\cdots<X_{a_{r}: n},
\end{aligned}
$$

where $X_{a_{i: n}}$ denotes $a_{i}^{\text {th }}$ observed failure time, $T$ is the predetermined experiment end time. The experiment is terminated at a random time $T^{*}=\max \left(X_{a_{r}: n}, T\right)$. The disadvantage of the multiply Type-II hybrid censoring scheme is excessively long experimental progressing time. So, we propose a generalized multiply Type-II hybrid censoring scheme in which the experiment is guaranteed to terminate 

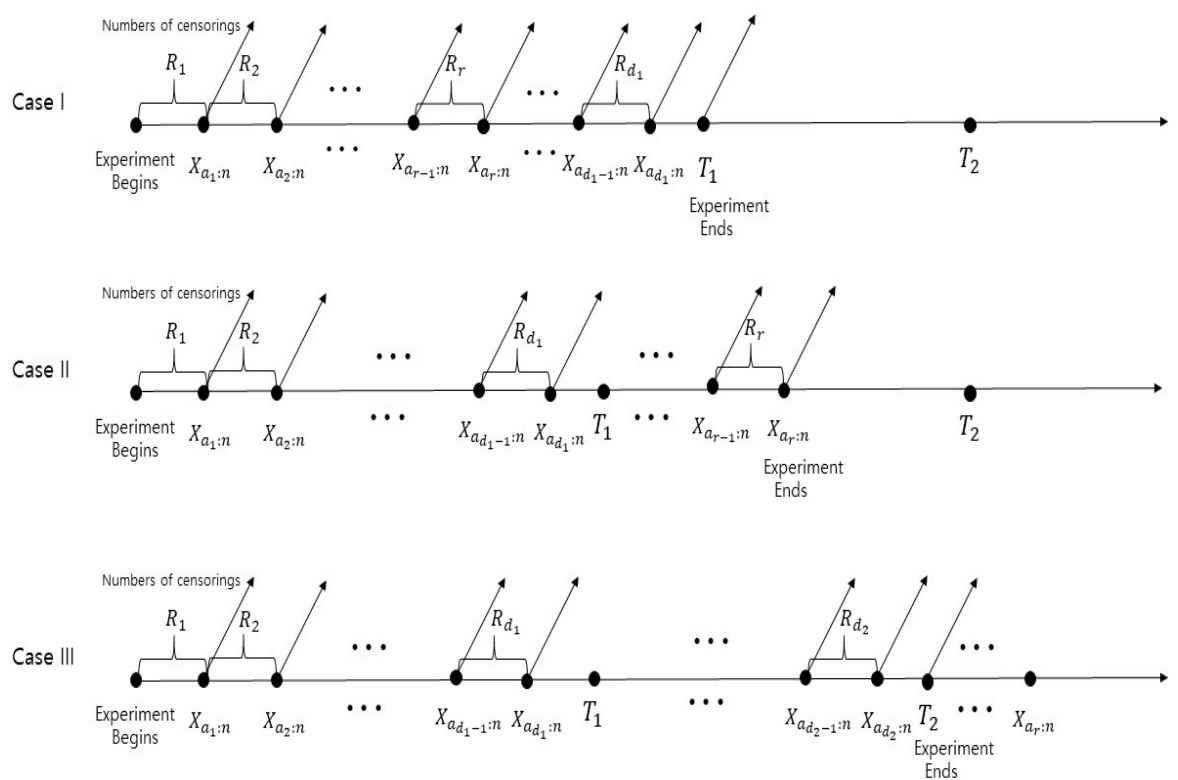

Figure 1: Generalized multiply Type-II hybrid censoring scheme.

at a pre-fixed extended experiment end time $T_{2}$. That is, the experiment is terminated at a random time $T^{*}=\min \left(\max \left(X_{a_{r}: n}, T_{1}\right), T_{2}\right)$. Let, $T_{1}$ is the predetermined experiment end time, $T_{2}$ is the extended experiment end time, $d_{i}(i=1,2)$ is the number of observed objects until $T_{i}(i=1,2)$, and $r$ is the predetermined observation number. A schematic representation of a generalized multiply Type-II hybrid censoring scheme is presented in Figure 1.

$$
\begin{aligned}
& \text { Case I : } X_{a_{1}: n}<X_{a_{2}: n}<\cdots<X_{a_{r}: n}<\cdots<X_{a_{d_{1}: n}}<T_{1} \text {, if } X_{a_{r: n}}<T_{1}, \\
& \text { Case II }: X_{a_{1}: n}<X_{a_{2}: n}<\cdots<X_{a_{d_{1}}: n}<T_{1}<\cdots<X_{a_{r}: n} \text { if } T_{1}<X_{a_{r: n}}<T_{2}, \\
& \text { Case III : } X_{a_{1}: n}<X_{a_{2}: n}<\cdots<T_{1}<\cdots<X_{a_{d_{2}: n}}<T_{2} \text {, if } T_{2}<X_{a_{r: n}} .
\end{aligned}
$$

Note that, $a_{r}=r+\sum_{i=1}^{r} R_{i}$, if $r+\sum_{i=1}^{r} R_{i}<n$ or $a_{r}=n$ if $r+\sum_{i=1}^{r} R_{i} \geq n$ when $n^{\text {th }}$ observation is not lost. $R_{i}=a_{i}-a_{i-1}-1$ and $a_{0}=0$. That is, $R_{i}$ is the unobserved or missing number between $X_{a_{i-1}: n}$ and $X_{a_{i}: n}$.

Then, the likelihood functions based on (2.2) are as follows.

$$
\begin{gathered}
\text { Case I : } L_{I}(\sigma) \propto \prod_{i=1}^{d_{1}} f\left(x_{a_{i}: n}\right) \prod_{i=1}^{d_{1}-1}\left[F\left(x_{a_{i+1}: n}\right)-F\left(x_{a_{i}: n}\right)\right]^{R_{i+1}} F\left(x_{a_{1}: n}\right)^{R_{1}}\left[1-F\left(T_{1}\right)\right]^{n-a_{d_{1}}}, \\
\text { Case II }: L_{I I}(\sigma) \propto \prod_{i=1}^{r} f\left(x_{a_{i}: n}\right) \prod_{i=1}^{r-1}\left[F\left(x_{a_{i+1}: n}\right)-F\left(x_{a_{i}: n}\right)\right]^{R_{i+1}} F\left(x_{a_{1}: n}\right)^{R_{1}}\left[1-F\left(x_{a_{r}: n}\right)\right]^{n-a_{r}}, \\
\text { Case III }: L_{I I I}(\sigma) \propto \prod_{i=1}^{d_{2}} f\left(x_{a_{i}: n}\right) \prod_{i=1}^{d_{2}-1}\left[F\left(x_{a_{i+1}: n}\right)-F\left(x_{a_{i}: n}\right)\right]^{R_{i+1}} F\left(x_{a_{1}: n}\right)^{R_{1}}\left[1-F\left(T_{2}\right)\right]^{n-a_{d_{2}}}
\end{gathered}
$$


We can rewrite the likelihood function by combining cases I, II, and III as follows.

$$
L \propto \prod_{i=1}^{m} f\left(x_{a_{i}: n}\right) \prod_{i=1}^{m-1}\left[F\left(x_{a_{i+1}: n}\right)-F\left(x_{a_{i}: n}\right)\right]^{R_{i+1}} F\left(x_{a_{1}: n}\right)^{R_{1}}[1-F(C)]^{n-a_{m}},
$$

where $C$ denotes the experiment end time, and $m$ is the observed number until the experiment end time $C$. The log-likelihood function is obtained by equations (1.1), (1.2), (2.4).

$$
\begin{aligned}
\ln L= & -m \ln \sigma-\frac{1}{\sigma} \sum_{i=1}^{m} x_{a_{i}: n}+\sum_{i=1}^{m-1} R_{i+1} \ln \left\{\exp \left(-\frac{x_{a_{i}: n}}{\sigma}\right)-\exp \left(-\frac{x_{a_{i+1}: n}}{\sigma}\right)\right\} \\
& +R_{1} \ln \left\{1-\exp \left(-\frac{x_{a_{1}: n}}{\sigma}\right)\right\}-\left(n-a_{m}\right) \frac{C}{\sigma} .
\end{aligned}
$$

On differentiating the log-likelihood (2.5) with respect to $\sigma$ and equation to zero, we obtain the estimating equation as follows.

$$
\begin{aligned}
\frac{\partial \ln L}{\partial \sigma}= & -\frac{1}{\sigma^{2}}\left[m \sigma-\sum_{i=1}^{m} x_{a_{i}: n}-\left(n-a_{m}\right) C+R_{1} \frac{x_{a_{1}: n} \exp \left(-\frac{x_{a_{1}: n}}{\sigma}\right)}{1-\exp \left(-\frac{x_{a_{1}: n}}{\sigma}\right)}\right. \\
& \left.-\sum_{i=1}^{m-1} R_{i+1} \frac{x_{a_{i}: n} \exp \left(-\frac{x_{a_{i}: n}}{\sigma}\right)-x_{a_{i+1}: n} \exp \left(-\frac{x_{a_{i+1}: n}}{\sigma}\right)}{\exp \left(-\frac{x_{a_{i}: n}}{\sigma}\right)-\exp \left(-\frac{x_{a_{i+1}: n}}{\sigma}\right)}\right]=0
\end{aligned}
$$

Equation (2.6) is complicated; therefore we cannot easily obtain the MLE. We then use the numerical technique in the following ways.

$$
\sigma=h(\sigma)
$$

where

$$
h(\sigma)=\frac{1}{m}\left[\sum_{i=1}^{m} x_{a_{i}: n}+\left(n-a_{m}\right) C-R_{1} \frac{x_{a_{1}: n} \exp \left(-\frac{x_{a_{1}: n}}{\sigma}\right)}{1-\exp \left(-\frac{x_{a_{1}: n}}{\sigma}\right)}+\sum_{i=1}^{m-1} R_{i+1} \frac{x_{a_{i}: n} \exp \left(-\frac{x_{a_{i}: n}}{\sigma}\right)-x_{a_{i+1}: n} \exp \left(-\frac{x_{a_{i+1}: n}}{\sigma}\right)}{\exp \left(-\frac{x_{a_{i}: n}}{\sigma}\right)-\exp \left(-\frac{x_{a_{i+1}: n}}{\sigma}\right)}\right] .
$$

We use a simple iterative scheme to solve equation (2.6) for $\sigma$. Start with an initial guess of $\sigma^{(0)}$, then obtain $\sigma^{(1)}=h\left(\sigma^{(0)}\right)$ and proceed in this way iteratively to obtain $\sigma^{(k)}=h\left(\sigma^{(k-1)}\right)$. Stop the iterative procedure when $\left|\sigma^{(k)}-\sigma^{(k-1)}\right|<\epsilon$, where $\epsilon$ is some pre-assigned tolerance limit. Then, we obtain the MLE $\tilde{\sigma}$ for $\sigma$.

\section{Bayesian estimation}

This section derives the Bayes estimators. To obtain the Bayes estimators, we have to select the prior distribution and the loss function. For the prior distribution, although using an informative prior is appropriate in the presence of sufficient information, a noninformative prior is preferable in its absence. Therefore, we consider two priors. First, a noninformative prior is as follows.

$$
\pi_{N}(\sigma) \propto \frac{1}{\sigma}
$$


Singh and Kumar (2007) obtained the Bayes estimators for the exponential distribution under a multiply Type-II censoring scheme. They used a generalized non-informative prior $1 / \sigma^{c}$. We consider $c=1$. By combining the likelihood function (2.4) and a noninformative prior (3.1), we obtain the posterior distribution $\pi_{N}(\sigma \mid \mathbf{x})$.

$$
\begin{aligned}
\pi_{N}(\sigma \mid \mathbf{x}) \propto & \sigma^{-m-1} \exp \left\{-\frac{1}{\sigma}\left(\sum_{i=1}^{m} x_{a_{i}: n}+\left(n-a_{m}\right) C\right)\right\} \\
& \times\left(1-\exp \left(-\frac{x_{a_{1}: n}}{\sigma}\right)\right)^{R_{1}} \prod_{i=1}^{m-1}\left\{\exp \left(-\frac{x_{a_{i}: n}}{\sigma}\right)-\exp \left(-\frac{x_{a_{i+1}: n}}{\sigma}\right)\right\}^{R_{i+1}}
\end{aligned}
$$

Second, an informative prior is as follows.

$$
\pi_{C}(\sigma) \propto \sigma^{-\alpha-1} \exp \left(-\frac{\beta}{\sigma}\right), \quad \sigma>0,
$$

where $\alpha, \beta>0$. The prior distribution (3.3) is known as the inversed gamma distribution. For $\beta=0$, $\pi_{C}(\sigma)$ is reduced to an improper prior. By combining the likelihood function (2.4) and an informative prior (3.3), we obtain the posterior distribution $\pi_{C}(\sigma \mid \mathbf{x})$.

$$
\begin{aligned}
\pi_{C}(\sigma \mid \mathbf{x}) \propto & \sigma^{-m-\alpha-1} \exp \left\{-\frac{1}{\sigma}\left(\sum_{i=1}^{m} x_{a_{i}: n}+\left(n-a_{m}\right) C+\beta\right)\right\} \\
& \times\left(1-\exp \left(-\frac{x_{a_{1}: n}}{\sigma}\right)\right)^{R_{1}} \prod_{i=1}^{m-1}\left\{\exp \left(-\frac{x_{a_{i}: n}}{\sigma}\right)-\exp \left(-\frac{x_{a_{i+1}: n}}{\sigma}\right)\right\}^{R_{i+1}} .
\end{aligned}
$$

Next, we consider the SELF, LLF, and GELF as the loss function. The SELF is commonly known as the symmetric loss function. The SELF is defined as $L_{1}(\sigma, \hat{\sigma})=(\hat{\sigma}-\sigma)^{2}$, with $\hat{\sigma}$ being an estimate of $\sigma$. Under the SELF, the Bayes estimator $\hat{\sigma}_{i S}(i=N, C)$ is obtained as the mean of the posterior distribution. That is,

$$
\hat{\sigma}_{i S}=E_{\sigma}(\sigma \mid \mathbf{x})=\frac{\int_{0}^{\infty} \sigma L(\sigma) \pi_{i}(\sigma) d \sigma}{\int_{0}^{\infty} L(\sigma) \pi_{i}(\sigma) d \sigma}, \quad i=N, C
$$

If $i=N$, the Bayes estimator is obtained by a noninformative prior and if $i=C$, the Bayes estimator is obtained by an informative prior. The symmetric loss function has equal weighting to the overestimation and underestimation of the same magnitudes. We consider two asymmetric loss functions because the overestimation is more serious in some situations than the underestimation (or vice-versa). The LLF is commonly known as the asymmetric loss function and defined as follows.

$$
L_{2}(\sigma, \hat{\sigma})=\exp (h(\hat{\sigma}-\sigma))-h(\hat{\sigma}-\sigma)-1, \quad h \neq 0,
$$

where the sign of parameter $h$ denotes the direction of asymmetry, and its magnitude reflects the degree of asymmetry. For $h<0$, the underestimation is more serious than the overestimation and for $h>0$, vice-versa. For $h$ close to zero, the LLF is approximately the SELF. By the LLF, we can obtain the Bayes estimator $\hat{\sigma}_{i L}(i=N, C)$ as follows.

$$
\hat{\sigma}_{i L}=-\frac{1}{h} \ln \left[E_{\sigma}(\exp (-h \sigma) \mid \mathbf{x})\right]=-\frac{1}{h} \ln \left[\frac{\int_{0}^{\infty} e^{-h \sigma} L(\sigma) \pi_{i}(\sigma) d \sigma}{\int_{0}^{\infty} L(\sigma) \pi_{i}(\sigma) d \sigma}\right], \quad i=N, C,
$$


if the above expectation exists. As another asymmetric loss function, we consider the GELF. The GELF is defined as follows.

$$
L_{3}(\sigma, \hat{\sigma}) \propto\left(\frac{\hat{\sigma}}{\sigma}\right)^{q}-q \ln \left(\frac{\hat{\sigma}}{\sigma}\right)-1, \quad q \neq 0 .
$$

For the shape parameter $q<0$, the underestimation is more serious than the overestimation and for $q>0$, vice-versa. For $q=-1$, the Bayes estimator obtained under the GELF coincides with the Bayes estimator obtained under the SELF. By the GELF, we can obtain the Bayes estimator $\hat{\sigma}_{i G}$ $(i=N, C)$ as follows.

$$
\hat{\sigma}_{i G}=\left[E_{\sigma}\left(\sigma^{-q} \mid \mathbf{x}\right)\right]^{-\frac{1}{q}}=\left[\frac{\int_{0}^{\infty} \sigma^{-q} L(\sigma) \pi_{i}(\sigma) d \sigma}{\int_{0}^{\infty} L(\sigma) \pi_{i}(\sigma) d \sigma}\right]^{-\frac{1}{q}}, \quad i=N, C .
$$

\subsection{Lindley's approximation}

In this subsection, we apply the Lindley's approximation method (Lindley, 1980) since equations (3.5), (3.7), and (3.9) cannot easily be solved. Lindley proposed the approximation method that can represent a simplified form of the Bayes estimator. Lindley's approximation method is as follows.

Consider the posterior expectation of a function $g(\sigma)$ given $\mathbf{x}$. That is,

$$
E(g(\sigma) \mid \mathbf{x})=\frac{\int g(\sigma) \exp (\ell(\sigma)+Q(\sigma)) d \sigma}{\int \exp (\ell(\sigma)+Q(\sigma)) d \sigma}
$$

where $g(\sigma)$ is function of $\sigma$ only, $\ell(\sigma)$ is the log-likelihood function and $Q(\sigma)$ is the log-prior distribution. Then, equation (3.10) can be approximated by

$$
E(g(\sigma) \mid \mathbf{x}) \approx g(\tilde{\sigma})+\hat{g}_{\sigma} \hat{Q}_{\sigma} \hat{\tau}_{\sigma \sigma}+\frac{1}{2} \hat{g}_{\sigma \sigma} \hat{\tau}_{\sigma \sigma}+\frac{1}{2} \hat{\ell}_{\sigma \sigma \sigma} \hat{\tau}_{\sigma \sigma}^{2} \hat{g}_{\sigma}
$$

where

$$
\begin{gathered}
\hat{g}_{\sigma}=\left.\frac{\partial g(\sigma)}{\partial \sigma}\right|_{\sigma=\tilde{\sigma}}, \hat{g}_{\sigma \sigma}=\left.\frac{\partial^{2} g(\sigma)}{\partial \sigma^{2}}\right|_{\sigma=\tilde{\sigma}}, \hat{Q}_{\sigma}=\left.\frac{\partial Q(\sigma)}{\partial \sigma}\right|_{\sigma=\tilde{\sigma}}, \\
\hat{\ell}_{\sigma \sigma \sigma}=\left.\frac{\partial^{3} \ell(\sigma)}{\partial \sigma^{3}}\right|_{\sigma=\tilde{\sigma}}, \hat{\ell}_{\sigma \sigma}=\left.\frac{\partial^{2} \ell(\sigma)}{\partial \sigma^{2}}\right|_{\sigma=\tilde{\sigma}}, \hat{\tau}_{\sigma \sigma}=-\left(\hat{\ell}_{\sigma \sigma}\right)^{-1} .
\end{gathered}
$$

Here, the estimator $\tilde{\sigma}$ denotes the MLE of $\sigma$. If the prior distribution is a noninformative prior (3.1), $\hat{Q}_{\sigma}=-1 / \tilde{\sigma}$ and if the prior distribution is an informative prior (3.3), $\hat{Q}_{\sigma}=-(\alpha+1) / \tilde{\sigma}+\beta / \tilde{\sigma}^{2}$. Now, we can obtain the Bayes estimators by approximating equations (3.5), (3.7) and (3.9). Under the SELF,

$$
g(\tilde{\sigma})=\tilde{\sigma}, \quad \hat{g}_{\sigma}=1, \quad \hat{g}_{\sigma \sigma}=0 .
$$

By substituting equation (3.12) into equation (3.11), the Bayes estimator $\hat{\sigma}_{i S}(i=N, C)$ is obtained as follows.

$$
\hat{\sigma}_{i S} \approx \tilde{\sigma}+\hat{Q}_{\sigma} \hat{\tau}_{\sigma \sigma}+\frac{1}{2} \hat{\ell}_{\sigma \sigma \sigma} \hat{\tau}_{\sigma \sigma}^{2}, \quad i=N, C
$$


Using equation (3.11), we can approximate the Bayes estimator $\hat{\sigma}_{i L}(i=N, C)$ obtained under the LLF.

$$
g(\tilde{\sigma})=\exp (-h \tilde{\sigma}), \quad \hat{g}_{\sigma}=-h \exp (-h \tilde{\sigma}), \quad \hat{g}_{\sigma \sigma}=h^{2} \exp (-h \tilde{\sigma}) .
$$

Equation (3.11) is substituted by equation (3.14) to obtain Bayes estimator $\hat{\sigma}_{i L}(i=N, C)$.

$$
\hat{\sigma}_{i L} \approx-\frac{1}{h} \ln L L, \quad i=N, C,
$$

where

$$
L L=e^{-h \tilde{\sigma}}-h e^{-h \tilde{\sigma}} \hat{Q}_{\sigma} \hat{\tau}_{\sigma \sigma}+\frac{h^{2}}{2} e^{-h \tilde{\sigma}} \hat{\tau}_{\sigma \sigma}-\frac{h}{2} e^{-h \tilde{\sigma}} \hat{\ell}_{\sigma \sigma \sigma} \hat{\tau}_{\sigma \sigma}^{2}
$$

Using equation (3.11), we can also approximate the Bayes estimator $\hat{\sigma}_{i G}(i=N, C)$ obtained under the GELF.

$$
g(\tilde{\sigma})=\tilde{\sigma}^{-q}, \quad \hat{g}_{\sigma}=-q \tilde{\sigma}^{-q-1}, \quad \hat{g}_{\sigma \sigma}=q(q+1) \tilde{\sigma}^{-q-2} .
$$

By substituting equation (3.16) into equation (3.11), the Bayes estimator $\hat{\sigma}_{i G}(i=N, C)$ is obtained as follows.

$$
\hat{\sigma}_{i G} \approx(L G)^{-\frac{1}{q}}, \quad i=N, C,
$$

where

$$
L G=\tilde{\sigma}^{-q}-q \tilde{\sigma}^{-q-1} \hat{Q}_{\sigma} \hat{\tau}_{\sigma \sigma}+\frac{q(q+1)}{2} \tilde{\sigma}^{-q-2} \hat{\tau}_{\sigma \sigma}-\frac{q}{2} \tilde{\sigma}^{-q-1} \hat{\ell}_{\sigma \sigma \sigma} \hat{\tau}_{\sigma \sigma}^{2}
$$

\subsection{Tierney-Kadane (T-K) approximation}

In this subsection, we consider another approximation method known as the T-K approximation (Tierney and Kadane, 1986). Let $L(\sigma)$ be the likelihood function of $\sigma$ based on $n$ observations, $\pi(\sigma)$ denotes the prior distribution of $\sigma$ and $\pi(\sigma \mid \mathbf{x})$ denotes the posterior distribution of $\sigma$. Then, we can write as follows.

$$
E[g(\sigma) \mid \mathbf{x}]=\int_{0}^{\infty} g(\sigma) \pi(\sigma \mid \mathbf{x}) d \sigma=\frac{\int_{0}^{\infty} e^{n l^{*}(\sigma)} d \sigma}{\int_{0}^{\infty} e^{n l(\sigma)} d \sigma}
$$

where

$$
l(\sigma)=\frac{1}{n}(\ln L(\sigma ; \mathbf{x})+\ln \pi(\sigma)), \quad l^{*}(\sigma)=\left(l(\sigma)+\frac{1}{n} \ln g(\sigma)\right) .
$$

Tierney and Kadane proposed the approximation method as follows;

$$
E[g(\sigma) \mid \mathbf{x}] \approx\left(\frac{\left|\psi^{*}\right|}{|\psi|}\right)^{\frac{1}{2}} \exp \left(n\left\{l^{*}\left(\hat{\sigma}^{*}\right)-l(\hat{\sigma})\right\}\right),
$$

where $\hat{\sigma}^{*}$ and $\hat{\sigma}$ maximize $l^{*}(\sigma)$ and $l(\sigma)$, respectively. $\psi^{*}$ and $\psi$ are minus the inverse of the second derivatives of $l^{*}(\sigma)$ and $l(\sigma)$ at $\hat{\sigma}^{*}$ and $\hat{\sigma}$, respectively. To use the T-K approximation method, we first 
obtain $l(\sigma)$ and $l^{*}(\sigma)$ using the log-likelihood function (2.5) and the prior distributions (3.1) and (3.3). In the case of a noninformative prior distribution (3.1),

$$
\begin{aligned}
l_{N}(\sigma)= & \frac{1}{n}\left(-(m+1) \ln \sigma-\frac{1}{\sigma}\left(\sum_{i=1}^{m} x_{a_{i}: n}+\left(n-a_{m}\right) C\right)\right. \\
& \left.+R_{1} \ln \left(1-\exp \left(-\frac{x_{a_{1}: n}}{\sigma}\right)\right)+\sum_{i=1}^{m-1} R_{i+1} \ln \left\{\exp \left(-\frac{x_{a_{i}: n}}{\sigma}\right)-\exp \left(-\frac{x_{a_{i+1}: n}}{\sigma}\right)\right\}\right), \\
l_{N}^{*}(\sigma)= & l_{N}(\sigma)+\frac{1}{n} \ln g(\sigma) .
\end{aligned}
$$

In the case of an informative prior distribution (3.3),

$$
\begin{aligned}
l_{C}(\sigma)= & \frac{1}{n}\left(-(m+\alpha+1) \ln \sigma-\frac{1}{\sigma}\left(\sum_{i=1}^{m} x_{a_{i}: n}+\left(n-a_{m}\right) C+\beta\right)\right. \\
& \left.+R_{1} \ln \left(1-\exp \left(-\frac{x_{a_{1}: n}}{\sigma}\right)\right)+\sum_{i=1}^{m-1} R_{i+1} \ln \left\{\exp \left(-\frac{x_{a_{i}: n}}{\sigma}\right)-\exp \left(-\frac{x_{a_{i+1}: n}}{\sigma}\right)\right\}\right), \\
l_{C}^{*}(\sigma)= & l_{C}(\sigma)+\frac{1}{n} \ln g(\sigma) .
\end{aligned}
$$

Now, we can obtain the Bayes estimators by the T-K approximation method. If the loss function is the SELF, $g(\sigma)=\sigma$. Then, we can obtain the Bayes estimator $\hat{\sigma}_{C S}$ by substituting equation (3.21) into equation (3.19).

$$
\begin{aligned}
\hat{\sigma}_{C S}= & \left(\frac{\left|\psi^{*}\right|}{|\psi|}\right)^{\frac{1}{2}}\left(\frac{\hat{\sigma}^{m+\alpha+1}}{\hat{\sigma}^{m+\alpha}}\right) \exp \left\{-\left(\frac{1}{\hat{\sigma}^{*}}-\frac{1}{\hat{\sigma}}\right)\left(\sum_{i=1}^{m} x_{a_{i}: n}+\left(n-a_{m}\right) C+\beta\right)\right\} \\
& \times\left(\frac{1-\exp \left(-\frac{x_{a_{1}: n}}{\hat{\sigma}^{*}}\right)}{1-\exp \left(-\frac{x_{a_{1}: n}}{\hat{\sigma}}\right)}\right)^{R_{1}} \prod_{i=1}^{m-1}\left(\frac{\exp \left(-\frac{x_{a_{i}: n}}{\hat{\sigma}^{*}}\right)-\exp \left(-\frac{x_{a_{i+1}: n}}{\hat{\sigma}^{*}}\right)}{\exp \left(-\frac{x_{a_{i}: n}}{\hat{\sigma}}\right)-\exp \left(-\frac{x_{a_{i+1}: n}}{\hat{\sigma}}\right)}\right)^{R_{i+1}} .
\end{aligned}
$$

If the loss function is the LLF, $g(\sigma)=e^{-h \sigma}$. Then, the Bayes estimator $\hat{\sigma}_{C L}$ is obtained by substituting equation (3.21) into equation (3.19) as follows.

$$
\hat{\sigma}_{C L}=-\frac{1}{h} \ln T L
$$

where

$$
\begin{aligned}
T L= & \left(\frac{\left|\psi^{*}\right|}{|\psi|}\right)^{\frac{1}{2}}\left(\frac{\hat{\sigma}}{\hat{\sigma}^{*}}\right)^{m+\alpha+1} \exp \left\{-\left(\frac{1}{\hat{\sigma}^{*}}-\frac{1}{\hat{\sigma}}\right)\left(\sum_{i=1}^{m} x_{a_{i}: n}+\left(n-a_{m}\right) C+\beta\right)-h \hat{\sigma}^{*}\right\} \\
& \times\left(\frac{1-\exp \left(-\frac{x_{a_{1}: n}}{\hat{\sigma}^{*}}\right)}{1-\exp \left(-\frac{x_{a_{1}: n}}{\hat{\sigma}}\right)}\right)^{R_{1}} \prod_{i=1}^{m-1}\left(\frac{\exp \left(-\frac{x_{a_{i}: n}}{\hat{\sigma}^{*}}\right)-\exp \left(-\frac{x_{a_{i+1}: n}}{\hat{\sigma}^{*}}\right)}{\exp \left(-\frac{x_{a_{i}: n}}{\hat{\sigma}}\right)-\exp \left(-\frac{x_{a_{i+1}: n}}{\hat{\sigma}}\right)}\right)^{R_{i+1}} .
\end{aligned}
$$


If the loss function is the GELF, $g(\sigma)=\sigma^{-q}$. Then, the Bayes estimator $\hat{\sigma}_{C G}$ is obtained by substituting equation (3.21) into equation (3.19) as follows.

$$
\hat{\sigma}_{C G}=(T G)^{-\frac{1}{q}},
$$

where

$$
\begin{aligned}
T G= & \left(\frac{\left|\psi^{*}\right|}{|\psi|}\right)^{\frac{1}{2}}\left(\frac{\hat{\sigma}^{m+\alpha+1}}{\hat{\sigma}^{*}{ }^{m+\alpha+1+q}}\right) \exp \left\{-\left(\frac{1}{\hat{\sigma}^{*}}-\frac{1}{\hat{\sigma}}\right)\left(\sum_{i=1}^{m} x_{a_{i}: n}+\left(n-a_{m}\right) C+\beta\right)\right\} \\
& \times\left(\frac{1-\exp \left(-\frac{x_{a_{1}: n}}{\hat{\sigma}^{*}}\right)}{1-\exp \left(-\frac{x_{a_{1}: n}}{\hat{\sigma}}\right)}\right)^{R_{1}} \prod_{i=1}^{m-1}\left(\frac{\exp \left(-\frac{x_{a_{i}: n}}{\hat{\sigma}^{*}}\right)-\exp \left(-\frac{x_{a_{i+1}: n}}{\hat{\sigma}^{*}}\right)}{\exp \left(-\frac{x_{a_{i}: n}}{\hat{\sigma}}\right)-\exp \left(-\frac{x_{a_{i+1}: n}}{\hat{\sigma}}\right)}\right)^{R_{i+1}} .
\end{aligned}
$$

By substituting $\alpha=0, \beta=0$ in equations (3.22), (3.23), and (3.24), we also obtain the Bayes estimators $\hat{\sigma}_{N i}(i=S, L, G)$.

\subsection{Markov chain Monte Carlo (MCMC) method}

In this subsection, we obtain the Bayes estimators by the MCMC method. The MCMC method is one of the best techniques for obtaining Bayes estimators. To obtain the MCMC samples from the two posterior distributions (3.2) and (3.4), we use the adaptive Metropolis-Hastings method and consider the normal distribution as the proposal distribution. However, the range of the random variable of the normal distribution is $(-\infty, \infty)$. Therefore, the MCMC samples are obtained as the $\ln \bar{\sigma}_{i j}(i=N, C)$. The $\log$-MLE $\ln \tilde{\sigma}$ as initial value is used.

Let, $\bar{\sigma}_{i j}$ denotes the $j^{\text {th }}$ the MCMC sample obtained by the two posterior distributions (3.2) and (3.4). If $i=N$, the MCMC samples are obtained by $\pi_{N}(\sigma \mid \mathbf{x})$ in (3.2), if $i=C$, the MCMC samples are obtained by $\pi_{C}(\sigma \mid \mathbf{x})$ in (3.4). NM is the number of the MCMC samples and $B$ is the burn-in period of the Markov chain. Then, we can obtain the Bayes estimators by the MCMC samples as follows.

$$
\begin{aligned}
& \hat{\sigma}_{i S}=E_{\sigma}(\sigma \mid \mathbf{x})=\frac{1}{N M-B} \sum_{j=B+1}^{N M} \bar{\sigma}_{i j}, \\
& \hat{\sigma}_{i L}=-\frac{1}{h} \ln E_{\sigma}\left(e^{-h \sigma} \mid \mathbf{x}\right)=-\frac{1}{h} \ln \frac{1}{N M-B} \sum_{j=B+1}^{N M} e^{-h \bar{\sigma}_{i j}}, \\
& \hat{\sigma}_{i G}=\left(E_{\sigma}\left(\sigma^{-q} \mid \mathbf{x}\right)^{-\frac{1}{q}}=\left(\frac{1}{N M-B} \sum_{j=B+1}^{N M} \bar{\sigma}_{i j}^{-q}\right)^{-\frac{1}{q}}, \quad i=N, C .\right.
\end{aligned}
$$

\section{Simulation results and illustration example}

\subsection{Simulation results}

In this subsection, we compare the simulated performance of all proposed estimators. We obtain the MSEs and biases for all proposed estimators through a Monte Carlo simulation with 5,000 replications. The simulation is performed with $\sigma=1$. To simulate, we take $\alpha=4$ and $\beta=3$ for the parameters of an informative prior distribution (3.3). For the LLF, the sign of parameter $h=-0.5$, 0.5. Likewise, the shape parameter $q=-0.5,0.5$ for the GELF. For an MCMC method, we obtain 
11,000 MCMC samples from each posterior distribution (3.2) and (3.4). 1,000 MCMC samples are used as the burn-in period of the MCMC. We also consider various versions of the generalized multiply Type-II hybrid censoring scheme. For example, the sample size $n=20(10) 40$, the predetermined experiment end time $T_{1}=1.0,1.3$ and the extended experiment end time $T_{2}=1.5,1.8$. The results are given in Tables $1-5$.

The Tables reveal that the MSEs generally decrease as the sample size $n$ increases. For the fixed sample size $n$, the MSEs generally decrease as the predetermined observation number $r$ increases. For the fixed sample size $n$ and the predetermined observation number $r$, the MSEs generally decrease as the predetermined experiment end time $T_{1}$ and the extended experiment end time $T_{2}$ increase. For the fixed sample size $n$, the predetermined observation number $r$ and the experiment end times $T_{1}, T_{2}$, the MSEs generally decrease as the number of multiply censored samples decreases.

Bayes estimators obtained by an informative prior are more efficient than the MLE in terms of MSE. When the sample size $n=20$, the best estimator is generally the estimator $\hat{\sigma}_{C L}$ obtained by the $h=0.5$ of the LLF and Lindley's approximation method. When the sample size $n=30,40$, the best estimator is generally the estimator $\hat{\sigma}_{C L}$ obtained by the $h=0.5$ of the LLF and MCMC samples in terms of MSE. For the fixed loss function, the Bayes estimators obtained by the MCMC samples and the Lindley approximation method of an informative prior are generally more efficient than the other estimators in terms of MSE, regardless of the loss function. For the fixed approximation method, the Bayes estimators obtained by $q=0.5$ of the GELF are generally more efficient than the other estimators except for the Bayes estimator obtained by using MCMC samples and an informative prior in terms of MSE.

\subsection{Real data}

Xia et al. (2009) reported a real-life dataset where the breaking strengths failure data of jute fiber are caused by two different gauge lengths $(10 \mathrm{~mm}$ and $20 \mathrm{~mm})$. We use the data with gauge length $10 \mathrm{~mm}$. The data are reported in Table 6.

These data are already used as the data for the exponential distribution by Saracoglu et al. (2012) and Wang and Li (2019); however, we check that the data follow the exponential distribution before analyzing the dataset. We obtain that the Kolmogorov-Smirnov distance is 0.17495 and the corresponding $p$-value is 0.2831 . To analyze the data, we have divided them by 100 for computational ease; however, this does not affect the inference. The applied results for the dataset are given in Tables 7-11.

\section{Conclusion}

The multiply Type-II hybrid censoring scheme has the disadvantage of an excessively long experimental time; therefore, we propose a generalized multiply Type-II hybrid censoring scheme, which ends by or before $T_{2}$. We also derive some estimators of the scale parameter of the exponential distribution under a generalized multiply Type-II hybrid censoring scheme. First, we obtain the MLE $\tilde{\sigma}$; second, we obtain the Bayes estimators by different prior distributions and loss functions. Since the Bayes estimators are directly difficult to obtain, we use three methods: Lindley's approximation, the $\mathrm{T}-\mathrm{K}$ approximation, and the MCMC sample. To compare the performance of all proposed estimators, we obtain their MSEs and biases through the Monte Carlo simulation, which reveals that the Bayes estimators obtained by an informative prior distribution have better performance than the other estimators. For the loss function, the Bayes estimators obtained by $q=0.5$ of the GELF generally have better performance than corresponding estimators obtained using the other loss functions. 


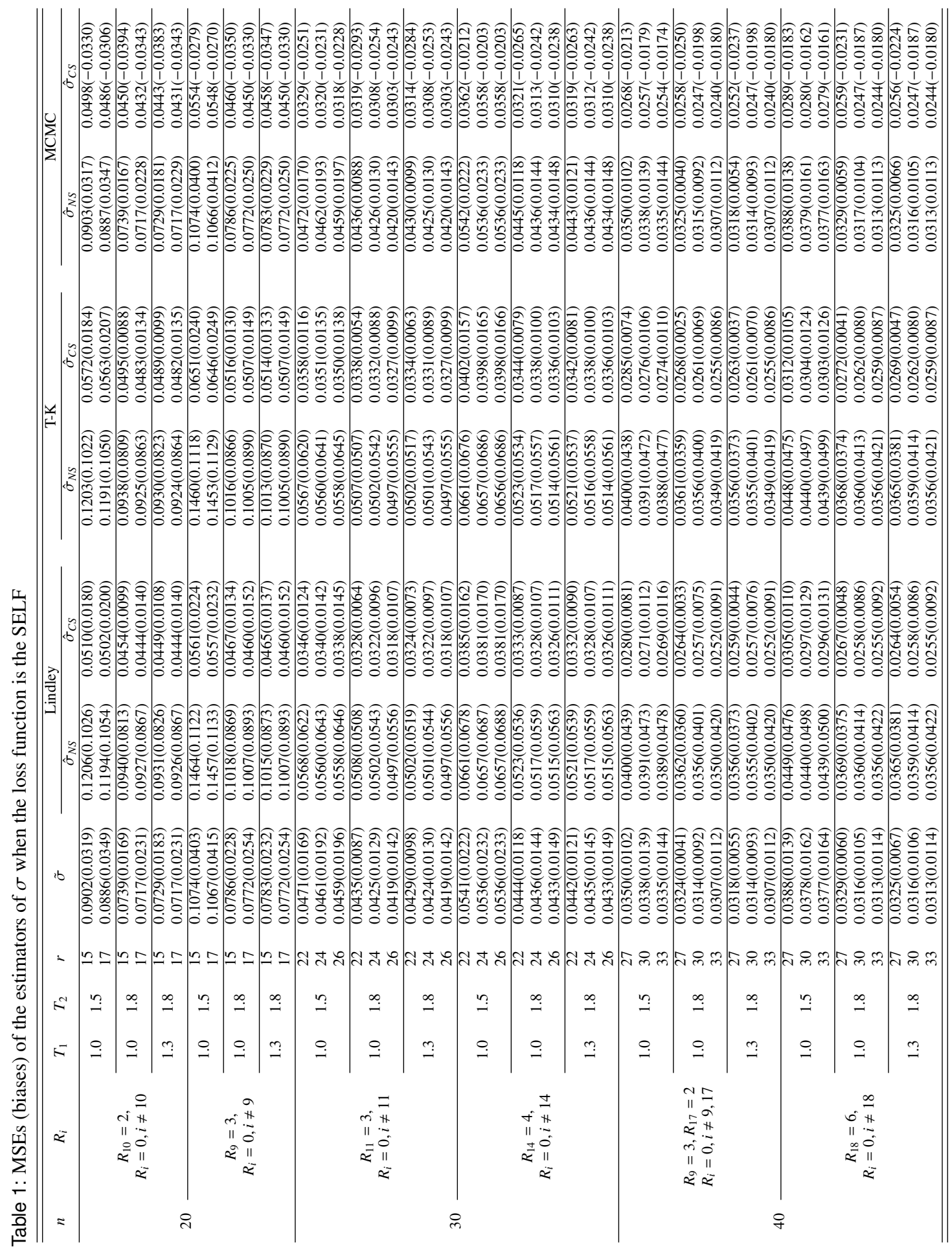




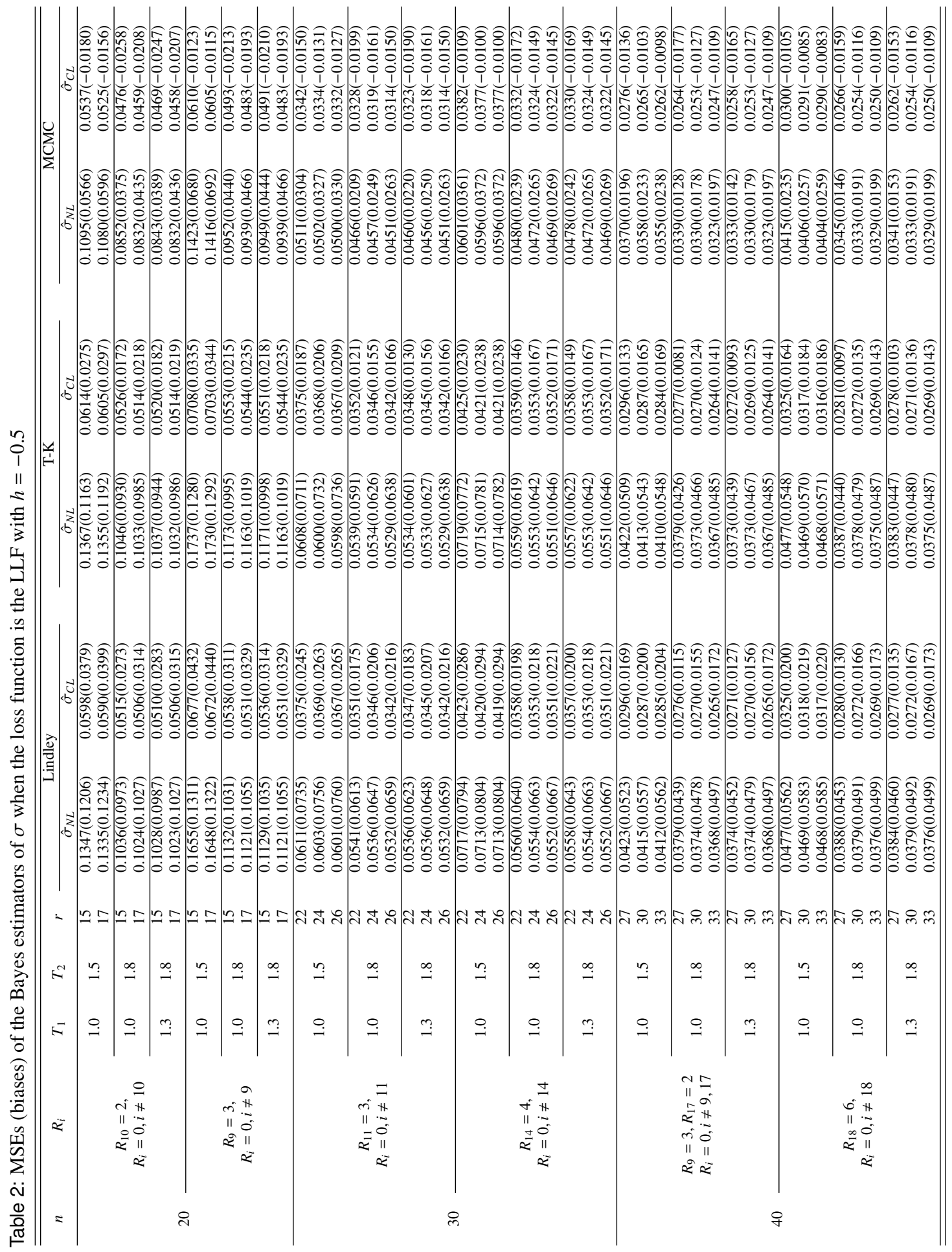




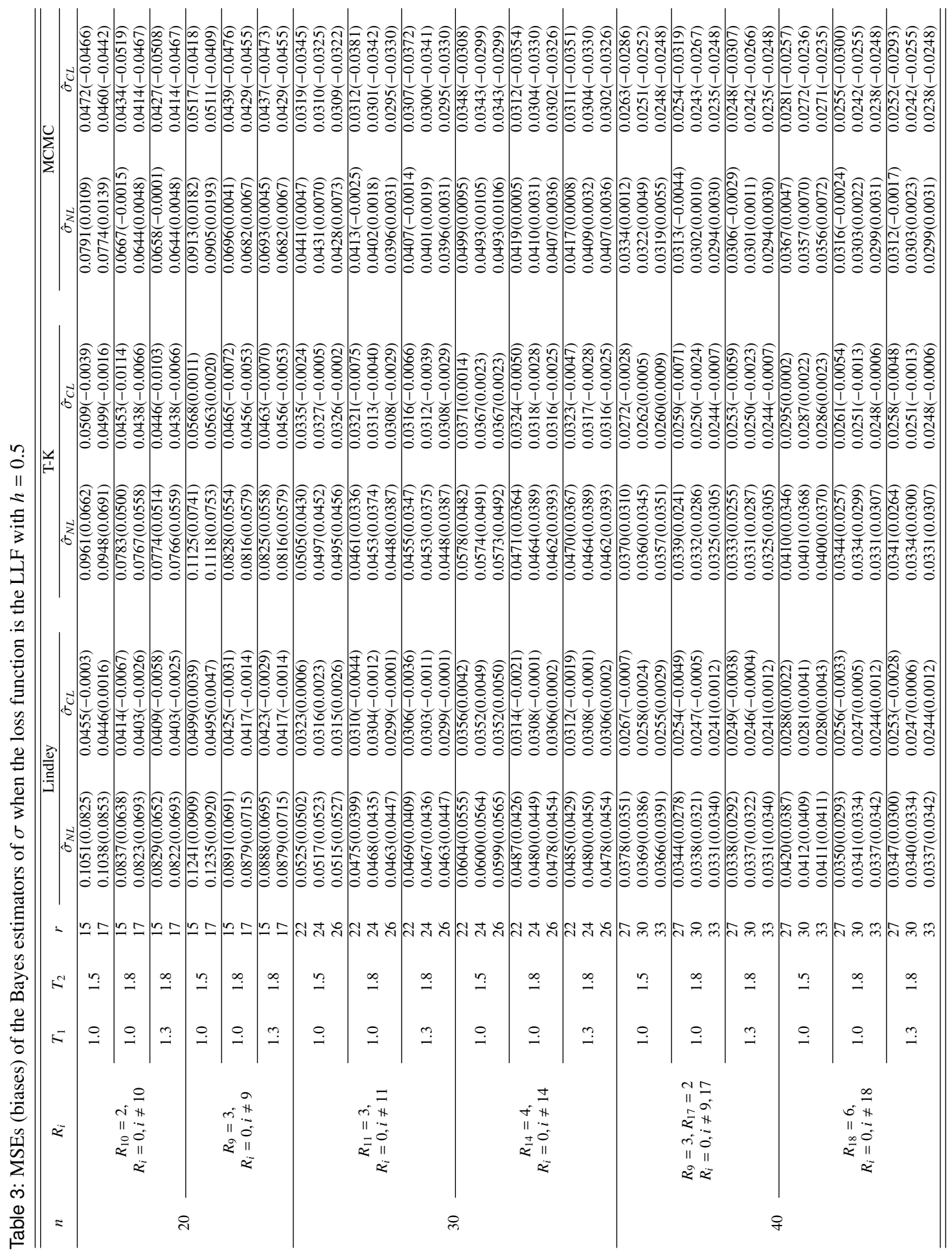




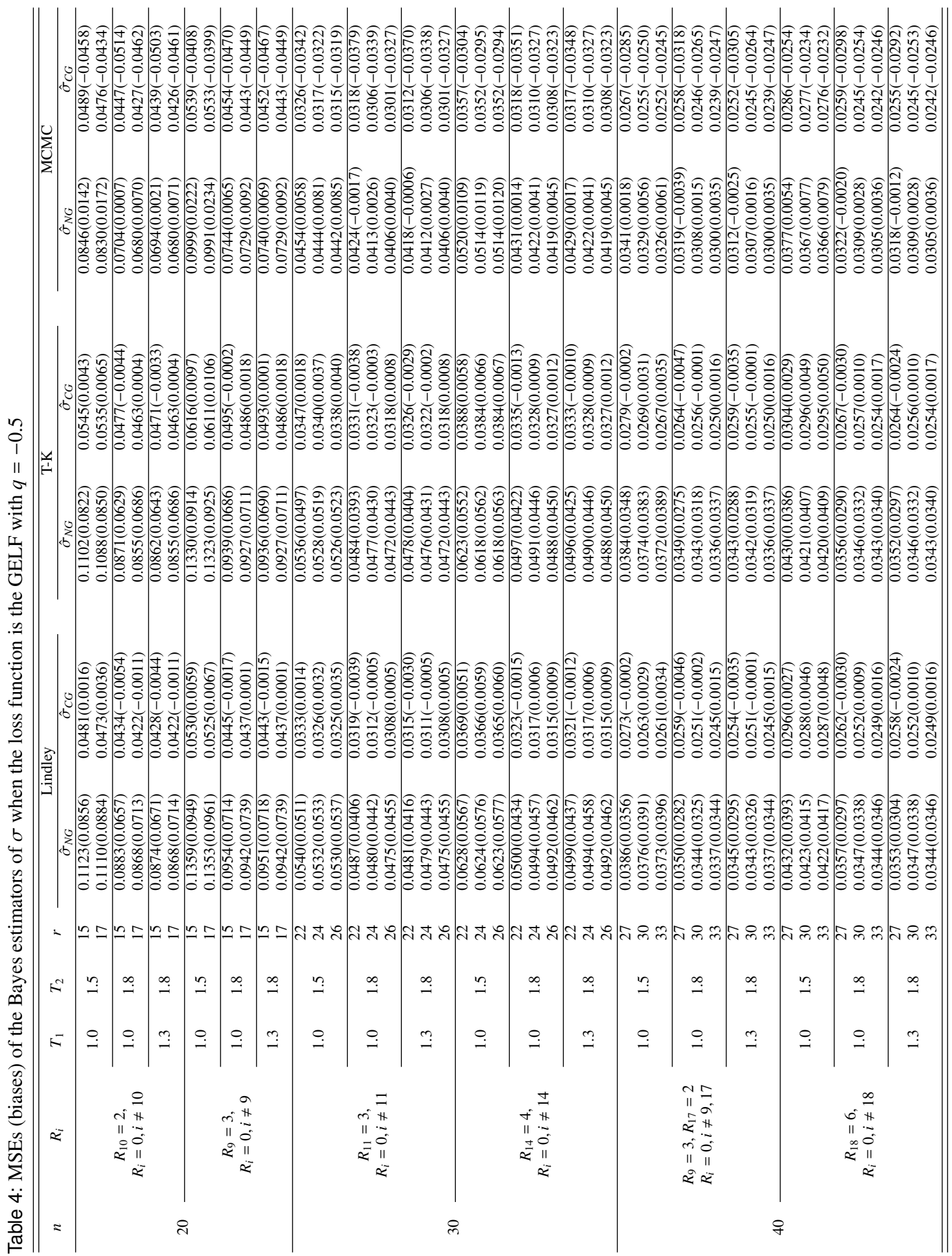




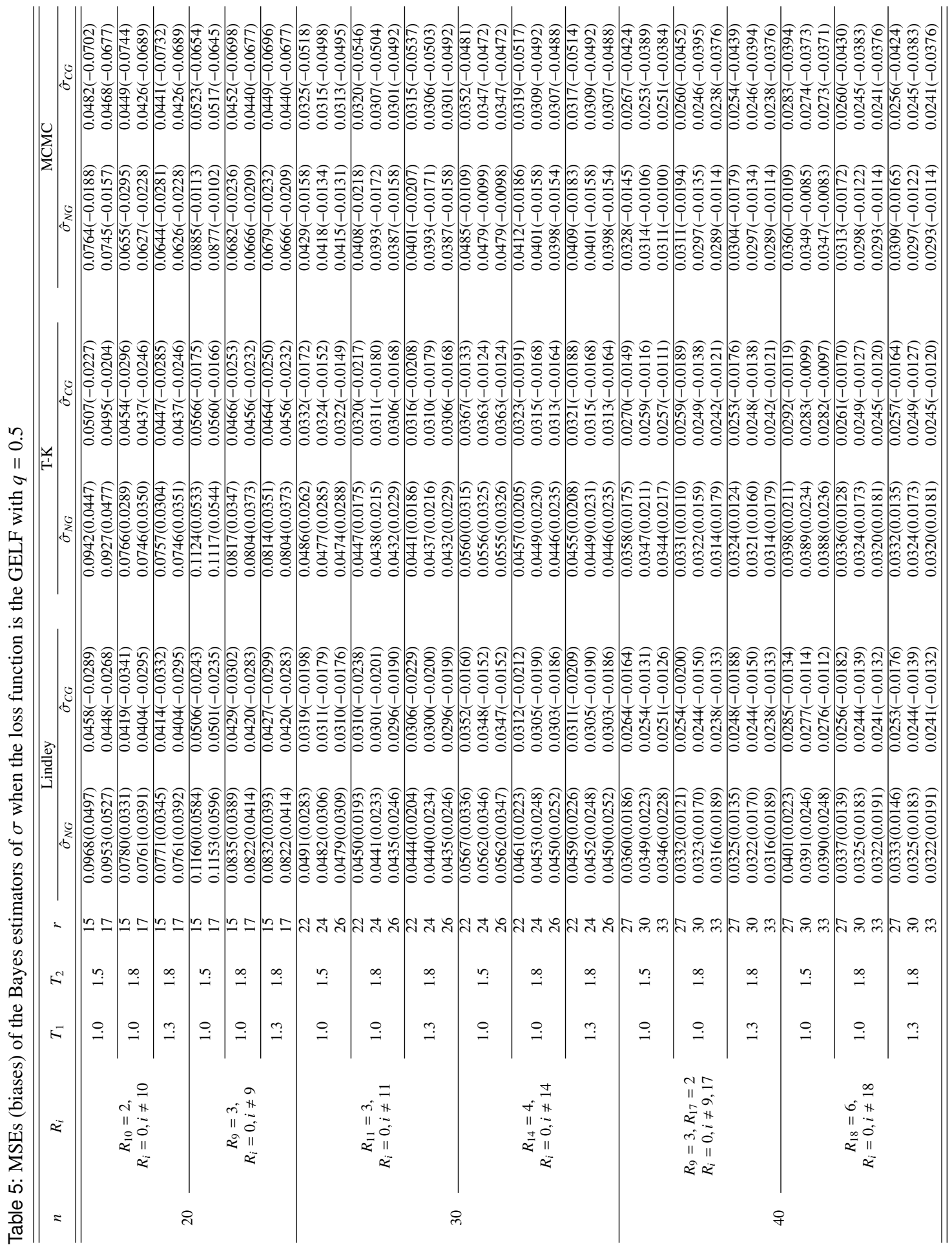


Table 6: Breaking strengths of jute fiber under 10mm gauge length

\begin{tabular}{rrrrrrrrrr}
\hline \hline 43.93 & 50.16 & 101.15 & 123.06 & 108.94 & 141.38 & 151.48 & 163.40 & 177.25 & 183.16 \\
212.13 & 257.44 & 262.90 & 291.27 & 303.90 & 323.83 & 353.24 & 376.42 & 383.43 & 422.11 \\
506.60 & 530.55 & 590.48 & 637.66 & 671.49 & 693.73 & 700.74 & 704.66 & 727.23 & 778.17 \\
\hline \hline
\end{tabular}

Table 7: Real data estimators when the loss function is the SELF

\begin{tabular}{|c|c|c|c|c|c|c|c|c|c|c|c|c|}
\hline \multirow{2}{*}{ Case } & \multirow{2}{*}{$n$} & \multirow{2}{*}{$R_{i}$} & \multirow{2}{*}{$T_{1}$} & \multirow{2}{*}{$T_{2}$} & \multirow{2}{*}{$r$} & \multirow{2}{*}{$\tilde{\sigma}$} & \multicolumn{2}{|c|}{ Lindley } & \multicolumn{2}{|c|}{$\mathrm{T}-\mathrm{K}$} & \multicolumn{2}{|c|}{ MCMC } \\
\hline & & & & & & & $\hat{\sigma}_{N S}$ & $\hat{\sigma}_{C S}$ & $\hat{\sigma}_{N S}$ & $\hat{\sigma}_{C S}$ & $\hat{\sigma}_{N S}$ & $\hat{\sigma}_{C S}$ \\
\hline I & & & & & 15 & 4.717 & 4.953 & 4.159 & 4.952 & 4.224 & 4.696 & 4.047 \\
\hline II & 30 & $R_{1}=2$ & 5 & 7 & 22 & 4.385 & 4.568 & 3.962 & 4.568 & 4.004 & 4.368 & 3.865 \\
\hline III & & $R_{i}=0, i \neq 1$ & & & 28 & 4.178 & 4.339 & 3.812 & 4.339 & 3.845 & 4.164 & 3.722 \\
\hline
\end{tabular}

SELF = squared error loss function; T-K = Tierney-Kadane; MCMC = Markov chain Monte Carlo. LLF = linex loss function; GELF = general entropy loss function

Table 8: Real data estimators when the loss function is the LLF with $h=-0.5$

\begin{tabular}{|c|c|c|c|c|c|c|c|c|c|c|c|}
\hline \multirow{2}{*}{ Case } & \multirow{2}{*}{$n$} & \multirow{2}{*}{$R_{i}$} & \multirow{2}{*}{$T_{1}$} & \multirow{2}{*}{$T_{2}$} & \multirow{2}{*}{$r$} & \multicolumn{2}{|c|}{ Lindley } & \multicolumn{2}{|c|}{ T-K } & \multicolumn{2}{|c|}{ MCMC } \\
\hline & & & & & & $\hat{\sigma}_{N L}$ & $\hat{\sigma}_{C L}$ & $\hat{\sigma}_{N L}$ & $\hat{\sigma}_{C L}$ & $\hat{\sigma}_{N L}$ & $\hat{\sigma}_{C L}$ \\
\hline I & & & & & 15 & 5.175 & 4.416 & 5.315 & 4.428 & 5.010 & 4.252 \\
\hline II & 30 & $R_{1}=2$ & 5 & 7 & 22 & 4.736 & 4.149 & 4.811 & 4.157 & 4.580 & 4.021 \\
\hline III & & $R_{i}=0, i \neq 1$ & & & 28 & 4.483 & 3.969 & 4.537 & 3.975 & 4.344 & 3.856 \\
\hline
\end{tabular}

Table 9: Real data estimators when the loss function is the LLF with $h=0.5$

\begin{tabular}{|c|c|c|c|c|c|c|c|c|c|c|c|}
\hline \multirow{2}{*}{ Case } & \multirow{2}{*}{$n$} & \multirow{2}{*}{$R_{i}$} & \multirow{2}{*}{$T_{1}$} & \multirow{2}{*}{$T_{2}$} & \multirow{2}{*}{$r$} & \multicolumn{2}{|c|}{ Lindley } & \multicolumn{2}{|c|}{$\mathrm{T}-\mathrm{K}$} & \multicolumn{2}{|c|}{ MCMC } \\
\hline & & & & & & $\hat{\sigma}_{N L}$ & $\hat{\sigma}_{C L}$ & $\hat{\sigma}_{N L}$ & $\hat{\sigma}_{C L}$ & $\hat{\sigma}_{N L}$ & $\hat{\sigma}_{C L}$ \\
\hline $\mathrm{I}$ & & & & & 15 & 4.675 & 4.018 & 4.645 & 4.031 & 4.457 & 3.890 \\
\hline II & 30 & $\begin{array}{l}R_{1}=2, \\
P\end{array}$ & 5 & 7 & 22 & 4.368 & 3.842 & 4.345 & 3.854 & 4.198 & 3.741 \\
\hline III & & $R_{i}=0, i \neq 1$ & & & 28 & 4.171 & 3.704 & 4.152 & 3.715 & 4.019 & 3.612 \\
\hline
\end{tabular}

LLF = linex loss function; T-K = Tierney-Kadane; MCMC = Markov chain Monte Carlo.

Table 10: Real data estimators when the loss function is the GELF with $q=-0.5$

\begin{tabular}{|c|c|c|c|c|c|c|c|c|c|c|c|}
\hline \multirow{2}{*}{ Case } & \multirow{2}{*}{$n$} & \multirow{2}{*}{$R_{i}$} & \multirow{2}{*}{$T_{1}$} & \multirow{2}{*}{$T_{2}$} & \multirow{2}{*}{$r$} & \multicolumn{2}{|c|}{ Lindley } & \multicolumn{2}{|c|}{ T-K } & \multicolumn{2}{|c|}{ MCMC } \\
\hline & & & & & & $\hat{\sigma}_{N G}$ & $\hat{\sigma}_{C G}$ & $\hat{\sigma}_{N G}$ & $\hat{\sigma}_{C G}$ & $\hat{\sigma}_{N G}$ & $\hat{\sigma}_{C G}$ \\
\hline I & & & & & 15 & 4.896 & 4.120 & 4.887 & 4.178 & 4.641 & 4.006 \\
\hline II & 30 & $R_{1}=2$ & 5 & 7 & 22 & 4.524 & 3.929 & 4.518 & 3.967 & 4.326 & 3.831 \\
\hline III & & & & & 28 & 4.300 & 3.781 & 4.296 & 3.812 & 4.127 & 3.691 \\
\hline
\end{tabular}

GELF = general entropy loss function; T-K = Tierney-Kadane; MCMC = Markov chain Monte Carlo.

Table 11: Real data estimators when the loss function is the GELF with $q=0.5$

\begin{tabular}{|c|c|c|c|c|c|c|c|c|c|c|c|}
\hline \multirow{2}{*}{ Case } & \multirow{2}{*}{$n$} & \multirow{2}{*}{$R_{i}$} & \multirow{2}{*}{$T_{1}$} & \multirow{2}{*}{$T_{2}$} & \multirow{2}{*}{$r$} & \multicolumn{2}{|c|}{ Lindley } & \multicolumn{2}{|c|}{ T-K } & \multicolumn{2}{|c|}{ MCMC } \\
\hline & & & & & & $\hat{\sigma}_{N G}$ & $\hat{\sigma}_{C G}$ & $\hat{\sigma}_{N G}$ & $\hat{\sigma}_{C G}$ & $\hat{\sigma}_{N G}$ & $\hat{\sigma}_{C G}$ \\
\hline I & & & & & 15 & 4.776 & 4.060 & 4.764 & 4.091 & 4.533 & 3.926 \\
\hline II & 30 & $\begin{array}{l}R_{1}=2, \\
R .-0 i+1\end{array}$ & 5 & 7 & 22 & 4.431 & 3.874 & 4.423 & 3.895 & 4.246 & 3.766 \\
\hline III & & $R_{i}=0, i \neq 1$ & & & 28 & 4.219 & 3.731 & 4.212 & 3.748 & 4.056 & 3.631 \\
\hline
\end{tabular}

GELF = general entropy loss function; T-K = Tierney-Kadane; MCMC = Markov chain Monte Carlo.

For the approximation method, the Bayes estimators obtained by the MCMC samples have generally better performance than corresponding estimators obtained by the other approximation methods.

This paper suffers from two limitations. First, we propose a generalized multiply Type-II hy- 
brid censoring scheme on the condition that the $n^{\text {th }}$ observation is not lost. For this reason, when $r+\sum_{i=1}^{r} R_{i} \geq n$, we can replace $a_{r}$ with $n$. The $n^{\text {th }}$ observation can be lost, in which case the last observation is not the $n^{\text {th }}$ observation. In reality, however, we have to progress until the $n^{\text {th }}$ observation to find the last observation when $r+\sum_{i=1}^{r} R_{i} \geq n$ under multiply censored samples. For example, when $n=20, r=16$, the $17^{\text {th }}$ failure occurs at present, but five units were censored, which prevents our observation of the predetermined observation number $r=16$. We are not sure whether the $17^{\text {th }}$ observation is the last one. To find the last observation, we have to continuously progress the experiment. Therefore, we have limited our scheme with the condition that the $n^{\text {th }}$ observation is not lost because no further observation can occur if the $n^{\text {th }}$ observation is not lost. Second, a noninformative prior may not be appropriate. We considered $1 / \sigma$ which is generally used as a noninformative prior since the expected fisher information can not easily be obtained. A noninformative prior is carefully selected and we will study more for choosing the appropriate noninformative.

\section{References}

Chan PS, Ng HKT, and Su F (2015). Exact likelihood inference for the two-parameter exponential distribution under Type-II progressively hybrid censoring, Metrika, 78, 747-770.

Chandrasekar B, Childs A, and Balakrishnan N (2004). Exact likelihood inference for the exponential distribution under generalized Type-I and Type-II hybrid censoring, Naval Research Logistics (NRL), 51, 994-1004.

Childs A, Balakrishnan N, and Chandrasekar B (2012). Exact distribution of the MLEs of the parameters and of the quantiles of two-parameter exponential distribution under hybrid censoring, Statistics, 46, 441-458.

Childs A, Chandrasekar B, Balakrishnan N, and Kundu D (2003). Exact likelihood inference based on Type-I and Type-II hybrid censored samples from the exponential distribution, Annals of the Institute of Statistical Mathematics, 55, 319-330.

Cho YS, Sun HK, and Lee KJ (2015). Exact likelihood inference for an exponential parameter under generalized progressive hybrid censoring scheme, Statistical Methodology, 23, 18-34.

Epstein B (1954). Truncated life tests in the exponential case, The Annals of Mathematical Statistics, 25, 555-564.

Ganguly A, Mitra S, Samanta D, and Kundu D (2012). Exact inference for the two-parameter exponential distribution under Type-II hybrid censoring, Journal of Statistical Planning and Inference, 142, 613-625.

Kang SB, Cho YS, Han JT, and Kim J (2012). An estimation of the entropy for a double exponential distribution based on multiply Type-II censored samples, Entropy, 14, 161-173.

Kang SB and Park SM (2005). Estimation for the exponentiated exponential distribution based on multiply Type-II censored samples, Communications for Statistical Applications and Methods, 12, 643-652.

Kang SB, Seo JI, and Kim YK (2013). Estimation for two-parameter generalized exponential distribution based on records, Communications for Statistical Applications and Methods, 20, 29-39.

Lee WH and Lee KJ (2018). Estimating the parameter of an exponential distribution under multiply Type-II hybrid censoring, Journal of the Korean Data and Information Science Society, 29, 807814.

Lindley DV (1980). Approximate Bayesian methods, Trabajos de estadistica y de investigacion operativa, 31, 223-245.

Saracoglu B, Kinaci I, and Kundu D (2012). On estimation of $R=P(Y<X)$ for exponential distri- 
bution under progressive Type-II censoring, Journal of Statistical Computation and Simulation, 82, 729-744.

Singh U and Kumar A (2007). Bayesian estimation of the exponential parameter under a multiply Type-II censoring scheme, Austrian Journal of Statistics, 36, 227-238.

Tierney L and Kadane JB (1986). Accurate approximations for posterior moments and marginal densities, Journal of the American Statistical Association, 81, 82-86.

Wang L and Li H (2019). Inference for exponential competing risks data under generalized progressive hybrid censoring, Communications in Statistics-Simulation and Computation, 1-17.

Xia ZP, Yu JY, Cheng LD, Liu LF, and Wang WM (2009). Study on the breaking strength of jute fibres using modified Weibull distribution, Composites Part A: Applied Science and Manufacturing, 40, $54-59$. 